\title{
IHMTC2017-12-1419
}

\section{Simulation OF RAREFIED GAS FLOW IN A RECTANGULAR ENCLOSURE}

\author{
Sumit Chamling Rai \\ h2016141044@hyderabad.bits-pilani.ac.in \\ K. Ram Chandra Murthy \\ rcmurthy@hyderabad.bits-pilani.ac.inc
}

\author{
Jayesh Sanwal \\ f20150440@hyderabad.bits-pilani.ac.in \\ Corresponding Author* \\ rcmurthy@hyderabad.bits-pilani.ac.inc
}

\begin{abstract}
The present work investigates the effects of rarefaction on gas flow patterns in a lid-driven cavity using the simulation package dsmcFoam, on the OpenFOAM platform. Direct Simulation Monte Carlo (DSMC) method is a simulation technique which caters to the regime in between the computationally intensive molecular dynamics solvers, as well as the often inaccurate $N S$ based solvers (applied to the rarefied gas simulations). It was proposed by G.A. Bird which employs the stochastic modelling of particle motion.

Simulations are performed and results are verified for the flow of a rarefied gas (Argon) for different lid velocities within the domain. The results are presented as streamlines, contours of velocity, pressure and temperature, along with velocities in $X$ and $Y$ directions. They have been found to be in good agreement with the previous experimental and numerical observations. Our simulations show that these eddies are much harder to observe in the rarefied domain, and cannot be observed upto velocities as high as $200 \mathrm{~m} / \mathrm{s}$ in a cavity with aspect ratio 1 .

Keywords: Rarefied gas flows, DSMC, Knudsen number, Cavity flow, Kinetic theory, Discrete methods.
\end{abstract}

\section{NOMENCLATURE}

Kn Knudsen number of the gas

$\lambda \quad$ Mean free path of the gas

L Characteristic length of the cavity

$N_{e q} \quad$ Number of equivalent particles

$N_{d s m c} \quad$ Number of DSMC particles inserted

$N_{\text {real }} \quad$ Number of real particles (explained in the outline)

$\mu \quad$ Dynamic Viscosity

$\mathrm{P}, P_{0} \quad$ Pressure, Reference Pressure (inside the cavity)

T, $T_{0} \quad$ Temperature, Reference Temperature

MD Molecular Dynamics

$V_{r m s} \quad$ Root mean square Velocity

AR Aspect Ratio

\section{INTRODUCTION}

Behavior of flows in extremely small sized domains is different from those in macroscopic size. A dimensionless constant, Knudsen Number, quantifies the degree of rarefaction of a gas and is defined as:

$$
K n=\frac{\lambda}{L}
$$

The conventional Navier-Stokes equation, which is based on the continuum assumption, is only applicable to flows when $\mathrm{Kn}$ $<0.1$ with slip boundary conditions at a solid wall. For Kn $>0.1$, the solution of the Navier-Stokes equation brings very large errors even with high order slip boundary conditions[3]. To analyze rarefied flows at high Knudsen numbers, multiple statistical mechanics models were developed. Among them, the Direct Simulation Monte Carlo (DSMC) method, pioneered by Bird G.A. (1976, 1994)[1] is often called the most effective numerical tool in simulating real engineering problems on a less computationally intensive environment. The DSMC method was proven to be consistent with the Boltzmann equation[7]. In hypersonic rarefied gas dynamics, DSMC has been proven to be an effective simulation method. With the development of MEMS and the further research on micro flows, DSMC method is now becoming a main numerical method in micro flow simulations. However, conventional DSMC method that is applied to hypersonic gas dynamics may not be directly used to simulate micro flows. The boundary conditions used in the conventional DSMC method is not appropriate for micro flows, which are usually subsonic flows. The present work investigates the flows of rarefied gases in these domains, at subsonic speeds, and aims to provide an accurate simulation based result.

\section{Outline of the DSMC method}

The DSMC method is a stochastic modelling of the Boltzmann equation. In this method, a large number of real particles are approximated to one single DSMC particle. The ratio real 
particles to DSMC particles is called $N_{e q}$ particles and is an input parameter for the simulation.

$$
N_{e q}=\frac{N_{\text {real }}}{N_{d s m c}}
$$

The velocity of these individual DSMC particles is then modelled using a Maxwell Boltzmann velocity distribution, and the $V_{r m s}$ is specified by the user. This defines the velocities of the particles stochastically, following which the meshing is done to discretize the domain. The interaction of the particles within each cell is then modelled for energy and momentum values, which are then obtained over the entire domain by collating the values of various cells.

$d s m c F o a m$ models particle-particle interactions within the cells, and their collisions with the domain boundaries. Since the DSMC method uses small cells within a domain, a time step is chosen such that it is much smaller than the mean collision time. For accurate results in OpenFOAM, time step is taken as the time taken by a DSMC particle at $V_{r m s}$ to cross close to one third of a cell. The DSMC method needs to specify the velocity, collision and wall interaction models to accurately capture the problem physics through existing models. Therefore the Larsen Bognarkke Variable Hard Sphere (VHS) model is used to model wall interactions, which was first modelled for the DSMC solutions in the original code by GA Bird [?, Bird, 1996] A No Time Counter (NTC) scheme is used to simulate the problem, along with a Monte Carlo (Maxwell distribution) velocity distribution, and a Maxwellian thermal wall interaction model.

In the DSMC method, the precise position of the particles at a given time step is of little significance, and physical quantities like pressure and temperature are extracted by averaging the particle motion over each cell. Therefore OpenFOAM uses time averaging of the contours for a certain number of timesteps, specified by the user. In the present study, time averaging of contours was done for 1000 time steps, to visualise macroscopic field quantities and their variations with time.

\section{PROBLEM DESCRIPTION}

The lid-driven cavity problem is a $2 \mathrm{D}$ flow problem with four adiabatic rigid walls, three of which are fixed, and the top wall moving with a constant velocity as shown in Fig 1. This problem has been simulated for different lid velocities as a benchmark problem in CFD for continuum flows. It has also been studied for the rarefied gas flows in the recent past.

We have studied this problem for different lid velocities at a constant Knudsen Number $(\mathrm{Kn}=1.06)$. The degree of rarefaction was controlled using the reference pressure, $P_{0}$. Knudsen number and pressure are related to each other through mean free path $(\lambda)$, defined as:

$$
\lambda=\frac{\mu}{p} \sqrt{\frac{\pi R T}{2}}
$$

where $P_{0}, T_{0}$ are reference pressure and temperature and $\mu$ is the dynamic viscosity.

\section{Boundary and Initial Conditions}

Top wall is adiabatic, moving with given finite velocity. The bottom and two side walls are adiabatic and stationary. The domain front and back walls are given symmetric boundary conditions, thus allowing no heat and mass flux across them. As an initial condition, temperature $T_{0}$ for all walls is set to $300 \mathrm{~K}$. The problem setup along with boundary conditions and mesh is shown in Fig. 1.

\section{Simulation setup}

The original DSMC95 algorithm given by GA Bird suggests taking a minimum of $20 \mathrm{dsmc}$ particles per cell, and the time step is taken such that a particle traveling at most probable velocity can cross not more than a third of the cell length. In accordance with this, simulations were run for 60 particles per cell, and a 2D domain with 100x100 divisions, yielding 10,000 cells. The time step was accordingly chosen as $2 \times 10^{-6} \mathrm{~s}$, and the size of the domain is $1 \mathrm{~m} \times 1 \mathrm{~m} \times 0.1 \mathrm{~m}$. Simulation were run for an end time of $1 \mathrm{~s}$, on a quad core Intel i5 $3.30 \mathrm{GHz}$ processor for different velocities of the lid, viz $10 \mathrm{~m} / \mathrm{s}, 50 \mathrm{~m} / \mathrm{s}, 100 \mathrm{~m} / \mathrm{s}$ and $200 \mathrm{~m} / \mathrm{s}$.

The same case was earlier run for coarser meshes of 40x40, and finer meshes up to $120 \times 120$. Mesh independence of sufficient degree was achieved on a 100x100 grid, and therefore that grid was used for all further simulations. Monoatomic Argon gas was used, and 501,728 dsmc particles were inserted into the domain, the $N_{e q}$ being $2.6 \times 10^{11}$ particles. The Knudsen number for all runs was 1.06 , while the Reynolds number varied. The reference particle diameter for the VHS model was taken as 4.17 $\mathrm{x} 10^{-10} \mathrm{~m}$.

\section{RESULTS AND DISCUSSION}

This study presents several interesting results, and gives insight into rarefied gas flows. The velocity (or momentum) streamlines overalapped on the pressure contours are as shown in Fig. 2. The plots show the movement of the gas inside the cavity, and significant observations can be made based on these.

The pressure plots clearly show that even for a velocity of $50 \mathrm{~m} / \mathrm{s}$, the pressure at the left side is much less than that on the right, with the difference being close to $1.9 \times 10^{-3}$, when compared to a average pressure in the domain of $5.5 \times 10^{-3}$ and the relative pressure differences become more pronounced by increasing the velocity of the lid further. This is the result of expansion of the gas due to the motion of the lid at the left, and compression at the right. This also implies that the pressure difference would increase on increasing the lid velocity, and viceversa, as can be seen in the cases of $200 \mathrm{~m} / \mathrm{s}$ (Fig. 5(e)) and $1 \mathrm{~m} / \mathrm{s}$ (Fig. 5(a)). The pressure difference for $200 \mathrm{~m} / \mathrm{s}$ is $9.5 \times 10^{-3}$ (about 170 percent) while for $1 \mathrm{~m} / \mathrm{s}$ it is $0.4 \times 10^{-3}$ (about 7 percent). The velocity streamlines also show that the motion of the gas is along the direction of motion of lid at the top, around $\mathrm{Y}=$ 0.7 to $\mathrm{Y}=1$. Due to the no slip wall effects, the velocity of gas at $\mathrm{Y}=1$ is equal to the lid velocity, and decreases rapidly. The $\mathrm{X}$-component of velocity decreases to zero and then increases in the opposite direction in the range $\mathrm{Y}=0$ to $\mathrm{Y}=0.7$. It is also observable that a vortex is formed, centred at $\mathrm{X}$ and around $\mathrm{Y}=$ 0.7. The length of the velocity vector increases within the domain with increase in lid velocity, along with larger difference in temperature across the wall as well. These results are shown in the form of the graphs plotted later (Fig. 3, 4 and 5). 


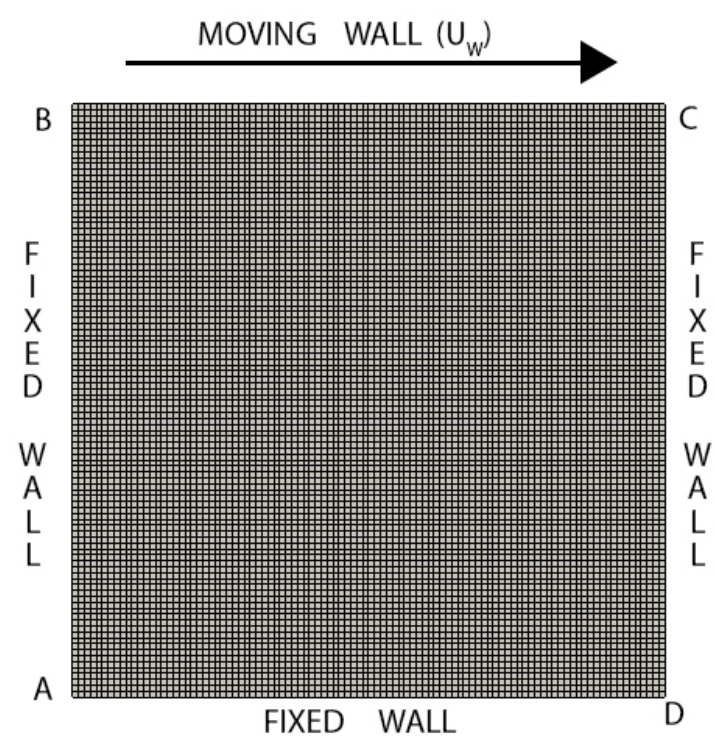

Figure 1. PROBLEM AND MESH GEOMETRY

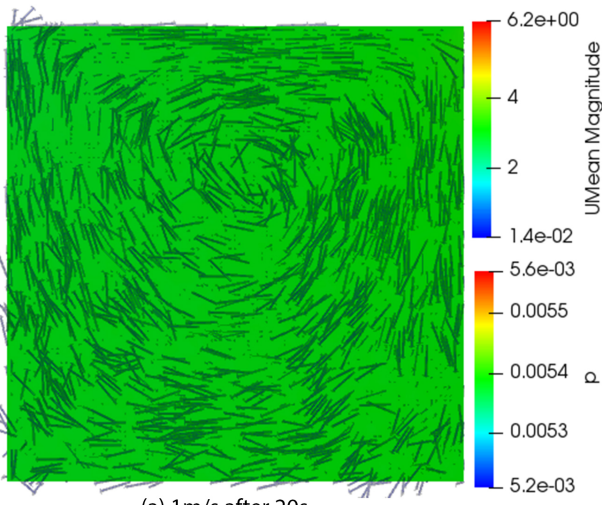

(a) $1 \mathrm{~m} / \mathrm{s}$ after $20 \mathrm{~s}$

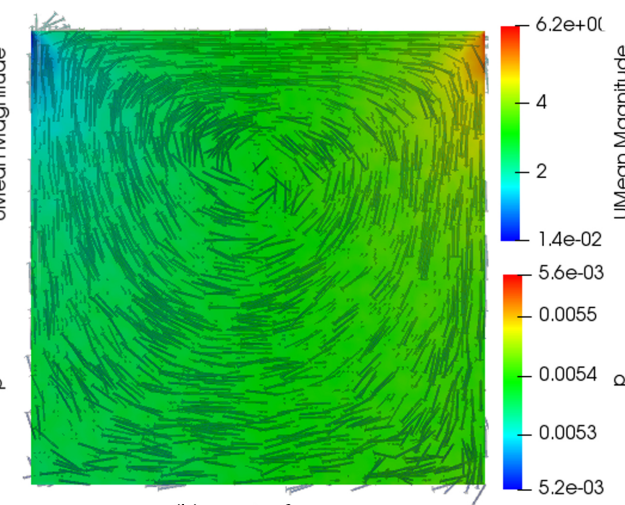

(b) $10 \mathrm{~m} / \mathrm{s}$ after $1 \mathrm{~s}$

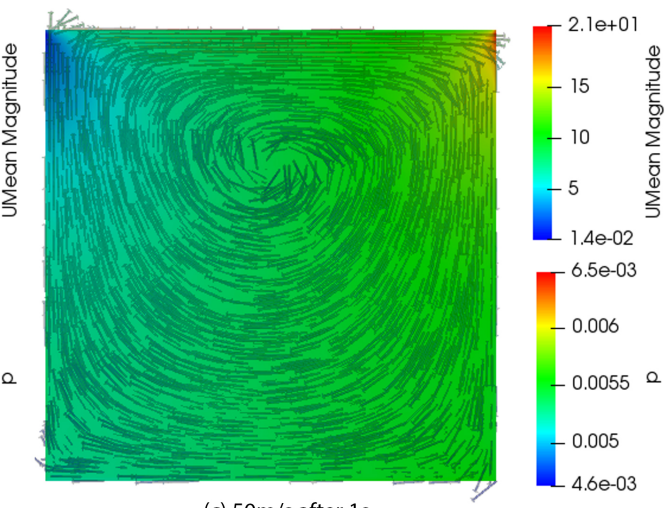

(c) $50 \mathrm{~m} / \mathrm{s}$ after $1 \mathrm{~s}$

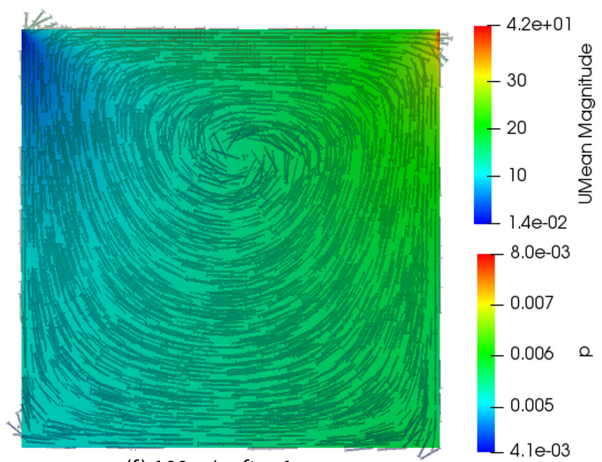

(f) $100 \mathrm{~m} / \mathrm{s}$ after $1 \mathrm{~s}$
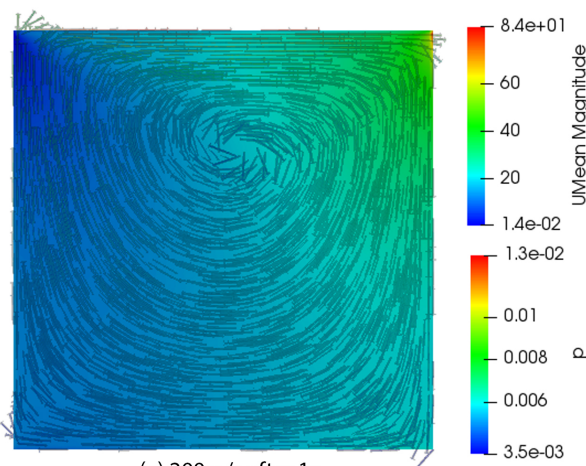

Figure 2. VARIATION OF VELOCITY OVERLAPPED ON PRESSURE AT (a) $1 \mathrm{~m} / \mathrm{s}$ (b) $10 \mathrm{~m} / \mathrm{s}$ (c) $50 \mathrm{~m} / \mathrm{s}$ (d) $100 \mathrm{~m} / \mathrm{s}$ (e) $200 \mathrm{~m} / \mathrm{s}$ 


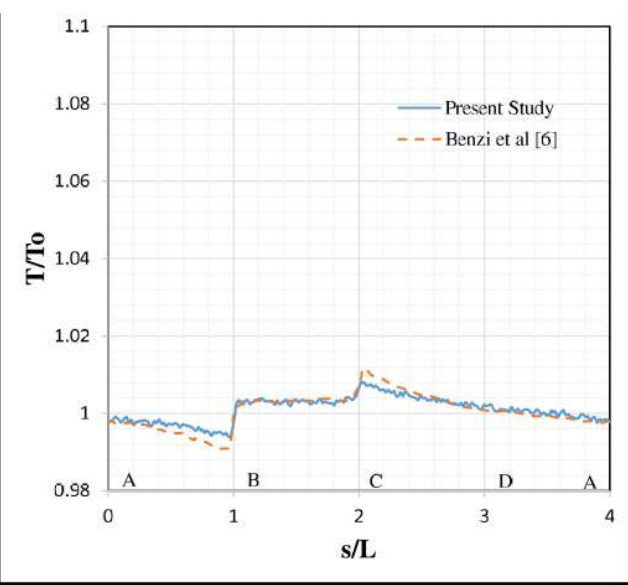

(a)

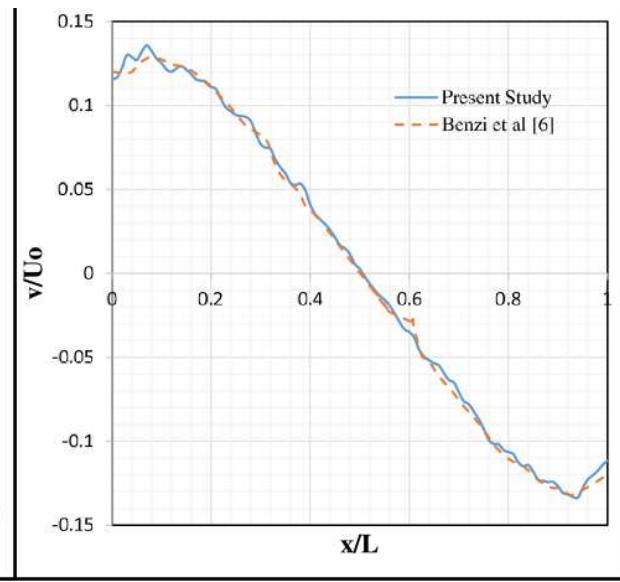

(b)

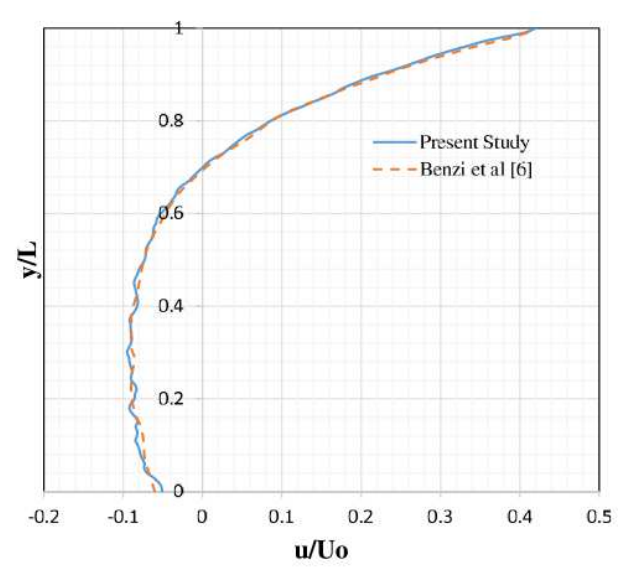

(c)

Figure 3. (a)VARIATION OF TEMPERATURE $\left(T / T_{0}\right)$ ALONG THE WALLS OF THE CAVITY (b) VARIATION OF $U / U_{0}$ ALONG X AXIS (c) VARIATION OF $Y / L$ ALONG $U / U_{0}$ AT $50 \mathrm{~m} / \mathrm{s}$ THROUGH VORTEX CENTER

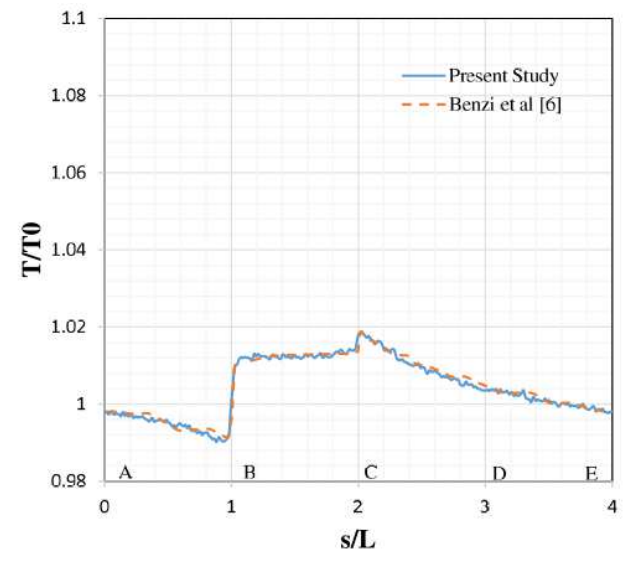

(a)

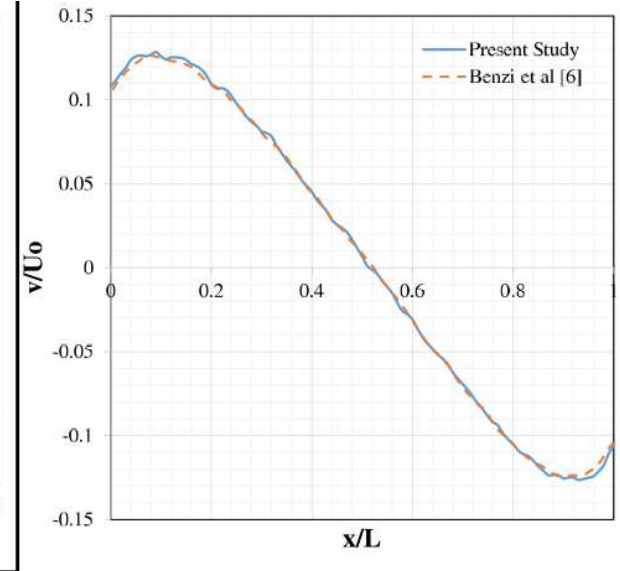

(b)

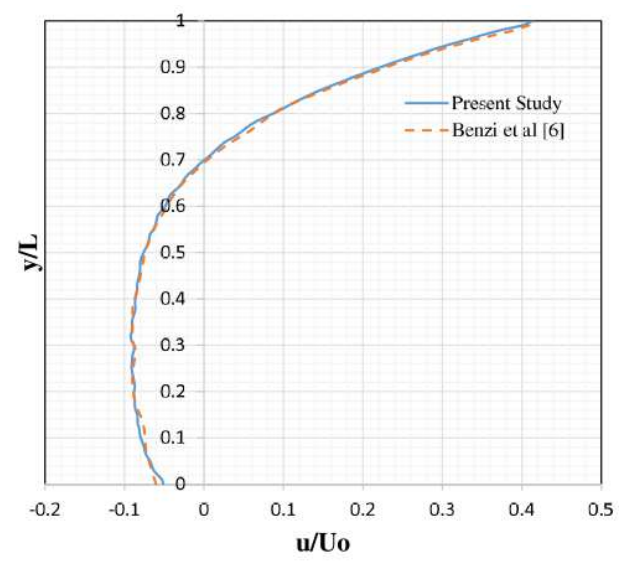

(c)

Figure 4. (a)VARIATION OF TEMPERATURE $\left(T / T_{0}\right)$ ALONG THE WALLS OF THE CAVITY (b) VARIATION OF $V / U_{0}$ ALONG X AXIS (c) VARIATION OF $Y / L$ ALONG $U / U_{0}$ AT $100 \mathrm{~m} / \mathrm{s}$

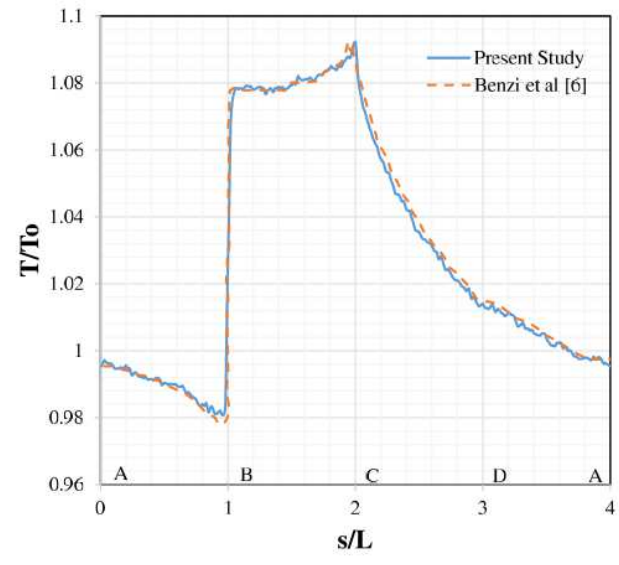

(a)

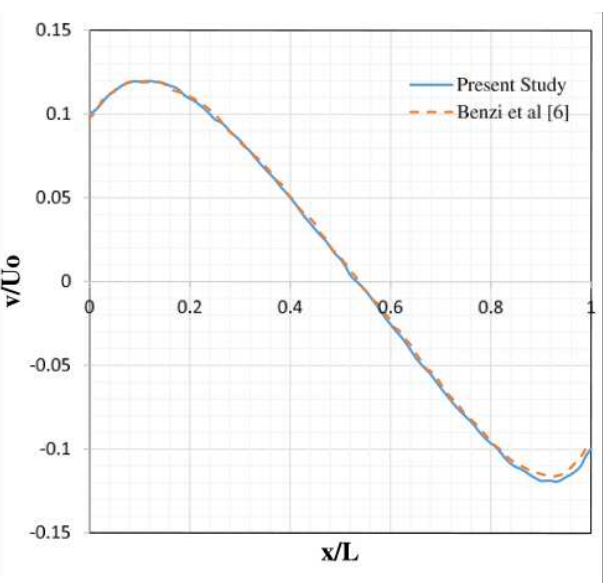

(b)

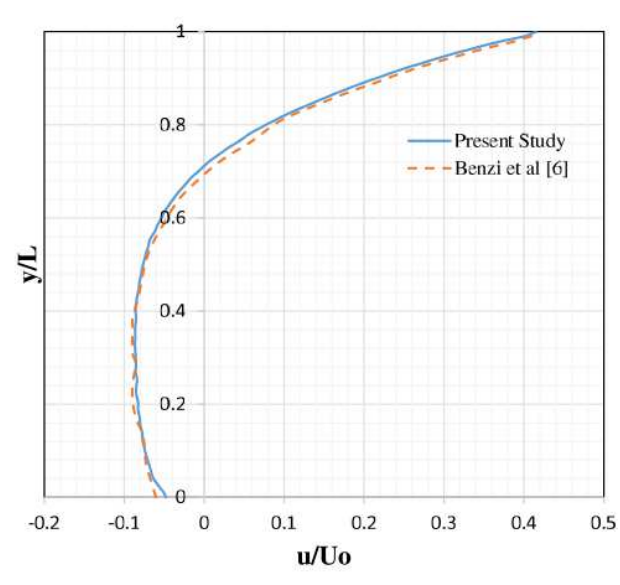

(c)

Figure 5. (a)VARIATION OF TEMPERATURE $\left(T / T_{0}\right)$ ALONG THE WALLS OF THE CAVITY (b) VARIATION OF $V / U_{0}$ ALONG $X$ AXIS (c) VARIATION OF $Y / L$ ALONG $U / U_{0}$ AT $200 \mathrm{~m} / \mathrm{s}$ 


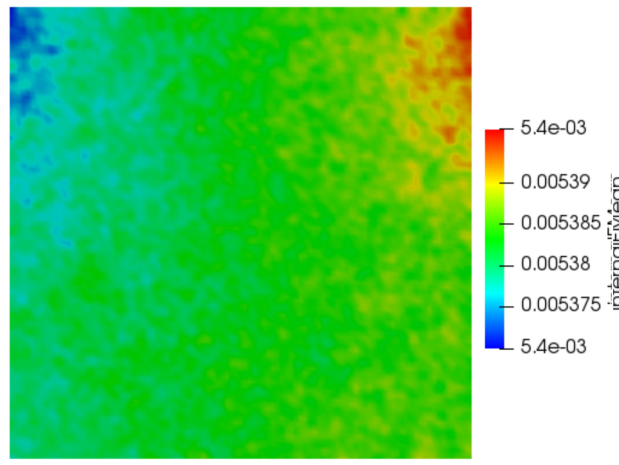

(a) $1 \mathrm{~m} / \mathrm{s}$ after $20 \mathrm{~s}$

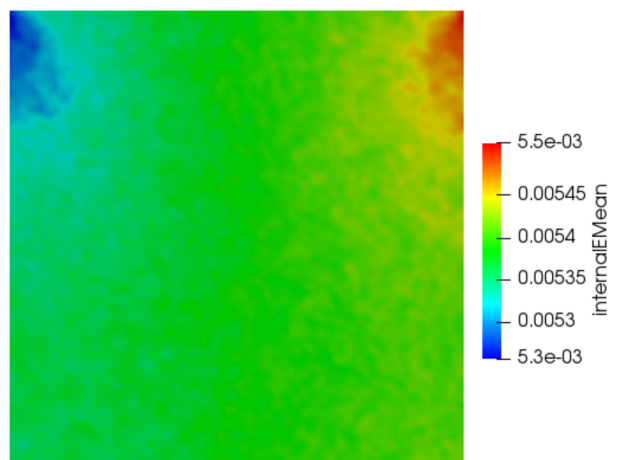

(b) $10 \mathrm{~m} / \mathrm{s}$ after $1 \mathrm{~s}$

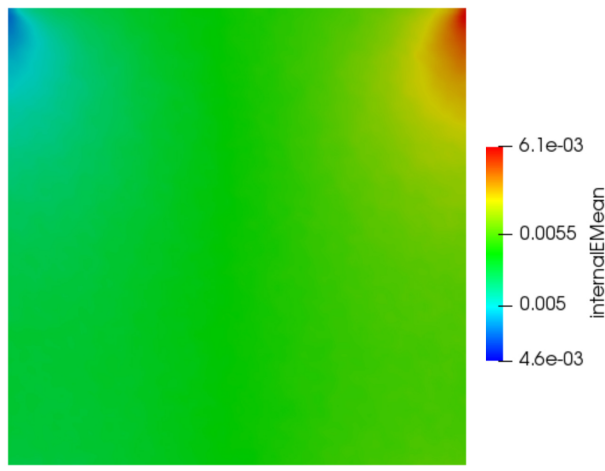

(c) $50 \mathrm{~m} / \mathrm{s}$ after $1 \mathrm{~s}$

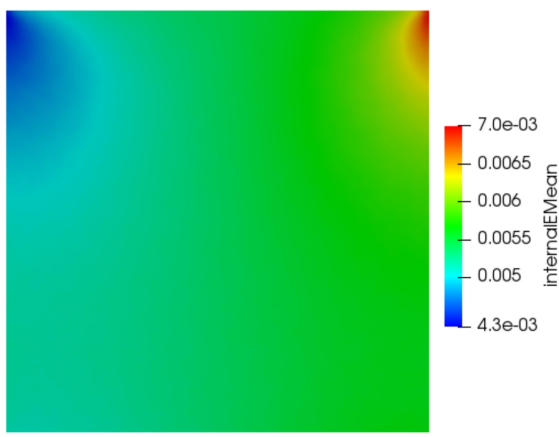

(f) $100 \mathrm{~m} / \mathrm{s}$ after $1 \mathrm{~s}$

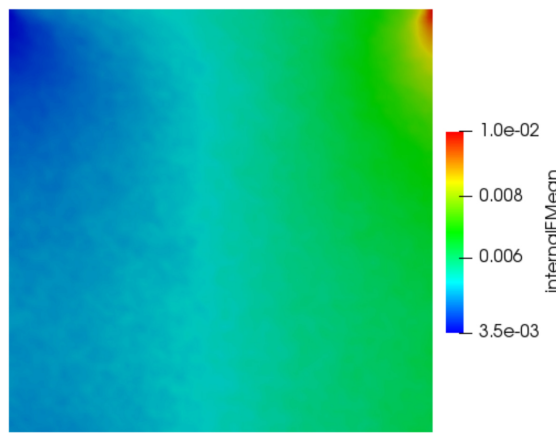

(e) $200 \mathrm{~m} / \mathrm{s}$ after $1 \mathrm{~s}$

Figure 6. VARIATION OF MEAN INTERNAL ENERGY (TEMPERATURE) OF THE CAVITY AT (a) $1 \mathrm{~m} / \mathrm{s}$ (b) $10 \mathrm{~m} / \mathrm{s}$ (c) $50 \mathrm{~m} / \mathrm{s}$ (d) $100 \mathrm{~m} / \mathrm{s}$ (e) $200 \mathrm{~m} / \mathrm{s}$

It is also interesting to note that even though the $\mathrm{X}$ component of mean velocity along a line parallel to the Y-axis does not change much on changing the lid velocity, the Y component of velocity changes plotted on a line parallel to the $\mathrm{X}$-axis changes significantly, ie a change of 12 percent is observable on either side. These plots were scaled by dividing values by $U_{0}$, thus using non-dimensional velocity to make the variables independent of lid velocity. These plots show us that the patterns remain largely independent of the lid velocity along the $\mathrm{Y}$ axis.

There are major differences between rarefied plots and plots in the continuum regime with similar lid velocities. In rarefied gases, the corner eddies are harder to observe, which is attributed to the lower density of the gas in the domain. Also, at the same Reynolds number of around 4, hardly any change in nondimensional velocity is observed in traditional incompressible solvers which has been widely documented. Another interesting trend is seen as the $\mathrm{Y}$ velocity along a line parallel to the $\mathrm{X}$-axis as shown in Fig 3(b), 4(b), 5(b). The curve shows that the magnitude of $\mathrm{Y}$ velocity first decreases to zero, with the minimum velocity occurring at $\mathrm{X}=0.5 \mathrm{~m}$. The graph is symmetric along $\mathrm{Y}=0.5 \mathrm{~m}$. Here, we can clearly see that the $\mathrm{Y}$-velocity rises to a maximum value first, around $\mathrm{X}=0.1 \mathrm{~m}$, and then after reaching a local maxima, decreases almost linearly from $X=0.1 \mathrm{~m}$ to $\mathrm{X}=0.9 \mathrm{~m}$, where it reaches its minimum value. After this, the velocity increases marginally from $X=0.9 \mathrm{~m}$ to $X=1 \mathrm{~m}$ ). The average of $\mathrm{z}$ momentum over $\mathrm{X}$ at every $\mathrm{Y}$ location is very close to zero. This shows that there is almost no average motion of DSMC particles along the Z-axis.

In Fig 3(a), 4(a), 5(a), we can clearly see from the graphs that the temperature along wall $\mathrm{AB}$ decreases, with a non linear rate of decrease. Along wall $\mathrm{BC}$, the initial temperature is higher than that in $\mathrm{AB}$, and increases to a maximum at $\mathrm{C}$. When moving from $\mathrm{C}$ to $\mathrm{D}$ along wall $\mathrm{CD}$, the temperature decreases rapidly, as can also be seen from the contours of the overall temperature distribution. The temperature continues to fall over wall DA, with the final temperature at A being slightly less than the reference temperature. Another notable observation is the variation of temperature with the lid velocity. We observe that by increasing the lid velocity, even though the overall trend of temperature variation remains the same, the graph is 'amplified' for higher lid velocities. The difference between the minimum and maximum values of $T / T_{0}$ (non-dimensional temperature) increases with increase in lid velocity.

\section{CONCLUSION}

The present study gives benchmarking results over the range of velocity for a given Knudsen number $(\mathrm{Kn}=1.06)$ and aspect ratio $(=1)$. We clearly see how the trends of various parameters change across the domain and verify the DSMC method using the dsmcFoam solver. The results are in accordance with the studies performed by various teams over the years, the most significant of which being the one conducted by John Benzi et al ${ }^{[6]}$.

We can conclude that the solver and the technique show promise, and can be used to study flows in microchannels as effectively as high speed flows. Further work on the field in this domain can deal with verification of other experimental results which are not yet studied in detail using the Direct Simulation Monte Carlo method, and can find greater applications in MEMS as well as high altitude aerodynamics. 


\section{REFERENCES}

1. In book, G.A. Bird, 1994. "Molecular Gas Dynamics and the Direct Simulation of Gas Flows". Oxford University Press.

2. In book, S. Chapman and T.G. Cowling, The Mathematical Theory of Non-Uniform Gas, 2nd Edition (1952), 3rd Edition (1970), Cambridge University Press.

3. In book, Ching Shen, 2005. "Rarefied gas flows Fundamental, Simulation and Microflows". Springer Publications.

4. In manual, OpenFoam Foundation. 2014-2017 "OpenFoam User Guide". CFD Direct.

5. In paper, Manuel Vargas, Giorgos Tatsios, 2014. "Rar- efied gas flow in a rectangular enclosure induced by nonisothermal walls", AIP: Physics of Fluids, 057101.

6. In paper, Benzi John, Xiao-Jun Gu, David R. Emerson, 2010. "Investigation Of Heat And Mass Transfer In a LidDriven Cavity Under Nonequilibrium Flow Conditions". Numerical Heat Transfer, Part B, 58: 287-303.

7. In paper, Wolfgang Wagner, February 1992. "A convergence proof for Bird's direct simulation Monte Carlo method for the Boltzmann equation". Journal of Statistical Physics, Volume 66, Issue 3-4, pg 1011-104. 